\title{
On the Characterizations of Chen's Two-Parameter Exponential Power Life-Testing Distribution
}

\author{
M. Shakil ${ }^{1}$, M. Ahsanullah ${ }^{2}$, and B. M. Golam Kibria ${ }^{3}$ \\ ${ }^{1}$ Miami Dade College, Hialeah, FL, USA \\ Rider University, Lawrenceville, New Jersey, USA \\ ${ }^{3}$ Florida International University, Miami, FL, USA
}

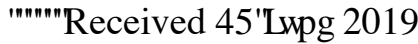

Accepted 0D) HUWU 2018

\begin{abstract}
Characterizations of probability distributions play important roles in probability and statistics. Before a particular probability distribution model is applied to fit the real world data, it is essential to confirm whether the given probability distribution satisfies the underlying requirements by its characterization. A probability distribution can be characterized through various methods. In this paper, we provide the characterizations of Chen's two-parameter exponential power life-testing distribution by truncated moment.
\end{abstract}

2010 Mathematics Subject Classifications: 60E05, 62E10, 62E15, 62G30

Keywords: Characterization, exponential power life-testing distribution, truncated moment

\section{Introduction}

As pointed out by Glänzel [8], the characterizations of probability distributions may serve as the basis for parameter estimations of a probability distributions. Before a particular probability distribution model is applied to fit the real world data, it is essential to confirm whether the given probability distribution satisfies the underlying requirements by its characterizations. Thus, characterizations of a probability distribution play important role in probability and statistics. A probability distribution can be characterized through various methods, see, for example, Ahsanullah et al. [3], and references therein. For an extensive survey on characterizations of univariate continuous distributions, the interested readers are referred to a recent monograph by Ahsanullah [2], and references therein.

In recent years, there has been a great interest in the characterizations of probability distributions by truncated moments. For example, the development of the general theory of the characterizations of probability distributions by truncated moment began with the work of Galambos and Kotz [7]. Further development on the characterizations of probability distributions 
by truncated moments continued with the contributions of many authors and researchers, among them Kotz and Shanbahag 11], Glänzel [8], and Glänzel et al. [9], are notable. However, most of these characterizations are based on a simple proportionality between two different moments truncated from the left at the same point. In this paper, we have considered a two-parameter exponential power life-testing distribution introduced by Chen [5], and provided its characterizations by truncated moment method. For other types of exponential power life-testing distributions and their various properties, studied by other authors and researchers, the interested readers are referred to Smith and Bain [16], Leemis [13], Rajarshi and Rajarshi [15], and Chen [4], among others.

The paper is organized as follows. In Section 2, the two-parameter exponential power lifetesting distribution introduced by Chen [5], and some of its properties are discussed. We present characterization results in Section 3. Finally, concluding remarks are presented in Section 4.

\section{Chen's Two-Parameter Exponential Power Life-Testing Distribution}

As introduced by Chen [5], a positive continuous random variable $X$ is said to have a twoparameter exponential power distribution with scale parameter $\lambda>0$ and shape parameter $\kappa>0$, which we will denote later as $X \sim$ chenexponentialpower $(\lambda, \kappa)$, if its probability density function is given by

$$
f(x)=\lambda k x^{k-1} e^{x^{\kappa}} e^{\lambda\left(1-e^{x^{k}}\right)}, k>0, \lambda>0 .
$$

The cumulative distribution function of $X \sim$ chenexponentialpower $(\lambda, \kappa)$ is given by

$$
F(x)=1-e^{\lambda\left(1-e^{x^{k}}\right)}, \quad x>0, k>0, \lambda>0 .
$$

For some selected values of the parameters, the graph of probability density function (2.1) and the cumulative distribution function (2.2) are illustrated in Figures 2.1 and 2.2 respectively. From these figures it appears that the proposed distribution is right skewed. 


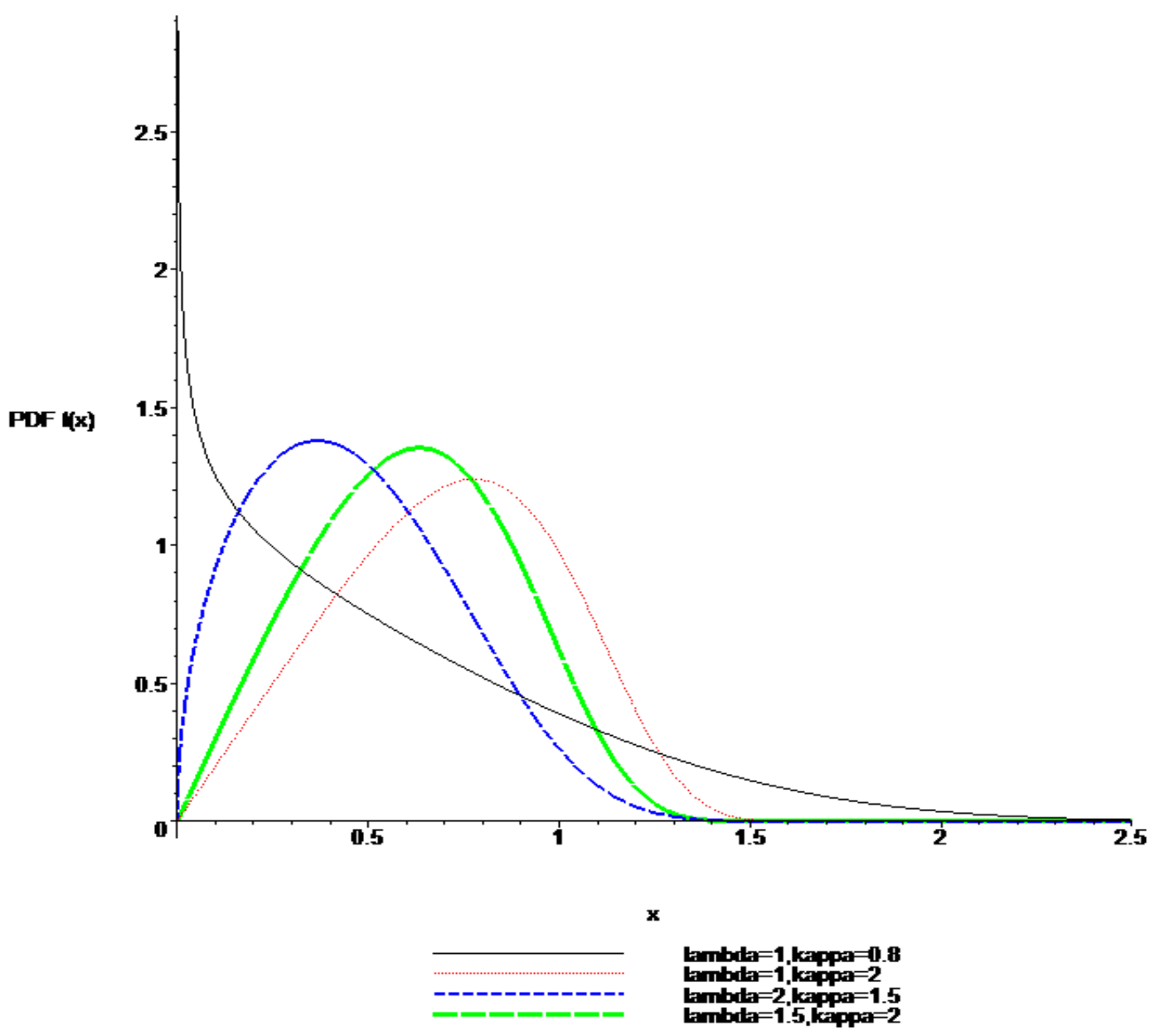

Figure 2.1. pdf, $f(x)$, when $X \sim$ chenexponentialpower $(\lambda, \kappa)$. 


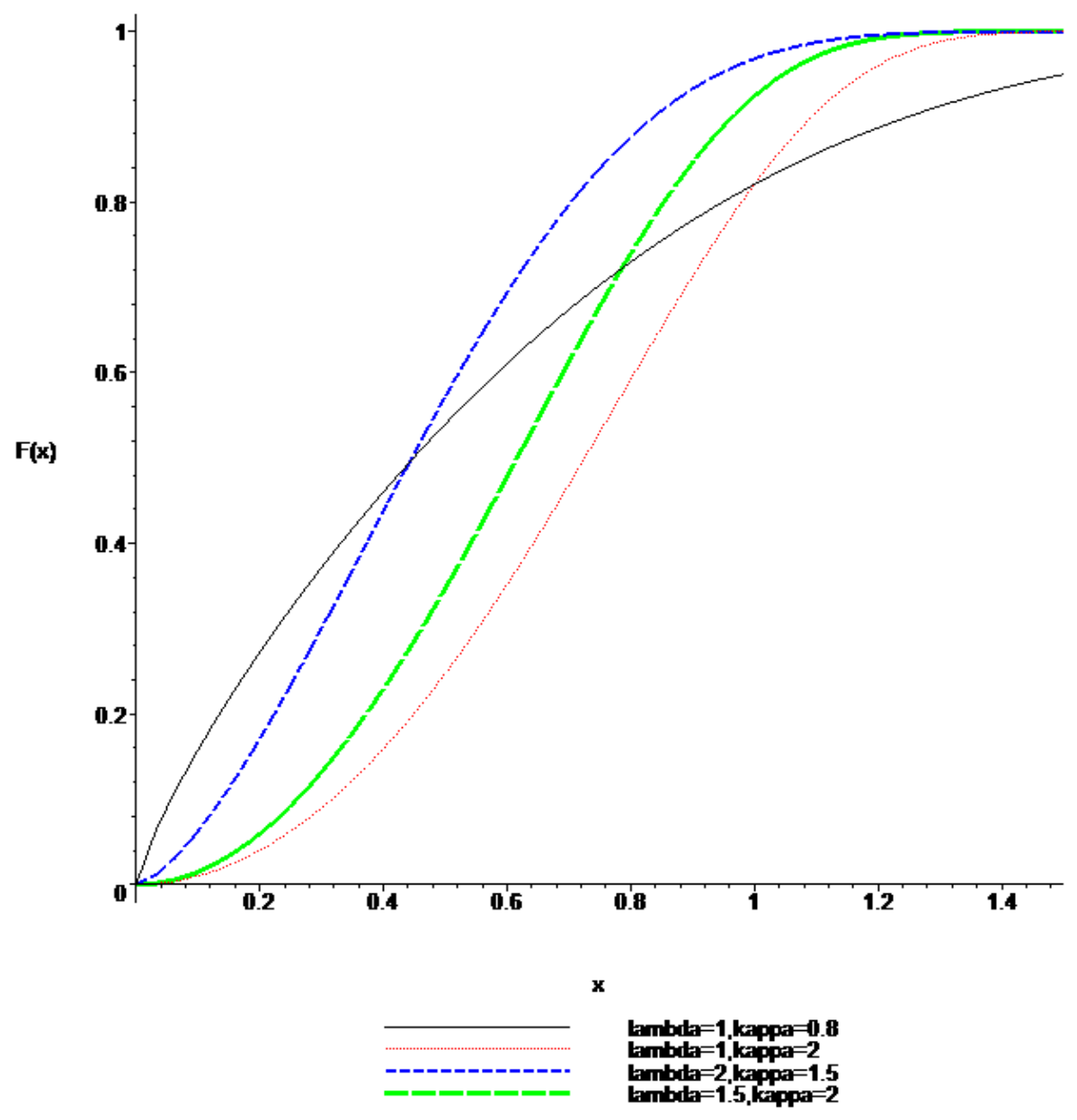

Figure 2.2. cdf, $F(x)$, when $X \sim$ chenexponentialpower $(\lambda, \kappa)$.

The corresponding survival (or reliability) and the hazard (or failure rate) functions, at any time, $x>0$, are respectively given by

$$
R(x)=1-F(x)=e^{\lambda\left(1-x^{\kappa}\right)},
$$

and

$$
h(x)=\frac{f(x)}{1-F(x)}=\lambda k x^{k-1} e^{x^{k}} .
$$

For some selected values of the parameters, the graph of hazard function (2.4) is illustrated in Figure 2.3 and it appears that hazard function has bathtub-shaped. 


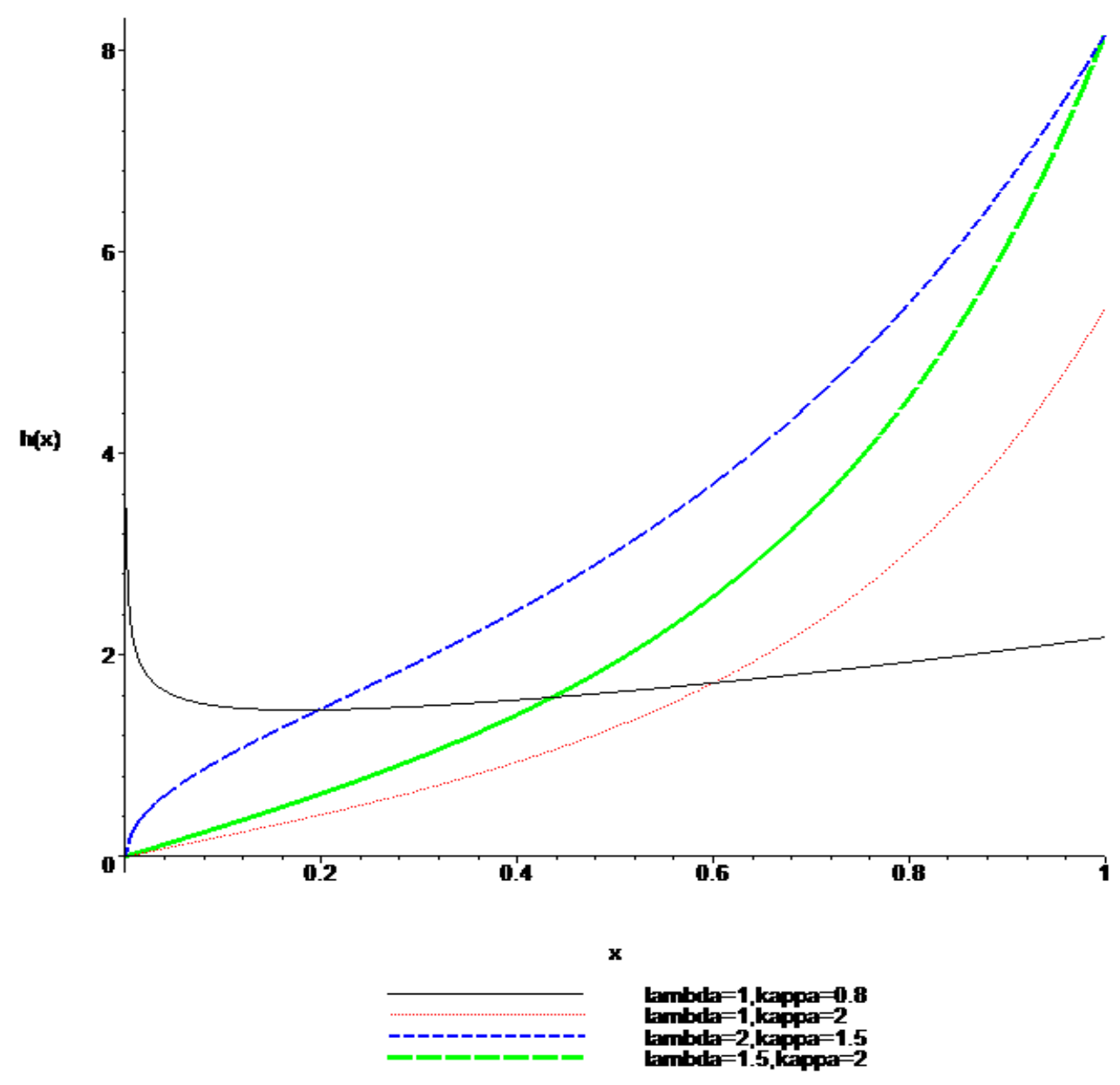

Figure 2.3. Hazard rate function, $h(x)$, when $X \sim$ chenexponentialpower $(\lambda, \kappa)$.

The $n t h$ moment, $E\left(X^{n}\right)$, where $n>0$ is an integer, is given by

$$
\begin{gathered}
E\left(X^{n}\right)=\int_{0}^{\infty} x^{n} f_{X}(x) d x \\
=\int_{0}^{\infty} x^{n} \lambda k x^{k-1} e^{x^{k}} e^{\lambda\left(1-e^{x^{k}}\right)} d x,
\end{gathered}
$$

which, on substituting $\lambda\left(1-e^{x^{\kappa}}\right)=u$, and simplifying, reduces to

$$
E\left(X^{n}\right)=-\int_{0}^{-\infty} e^{u}\left[\ln \left(1-\frac{u}{\lambda}\right)\right]^{\frac{n}{k}} d u
$$


Letting $u=-t \quad$ in $\quad(2.5), \quad$ and simplifying, we have

$$
E\left(X^{n}\right)=\int_{0}^{\infty} e^{-t}\left[\ln \left(1+\frac{t}{\lambda}\right)\right]^{\frac{n}{k}} d t
$$

It is obvious from (2.6) that the 1 st moment, $E(X)$, is mathematically easily tractable for $k=1$. So, by taking $n=1, k=1$ in (2.6), using Gradshteyn and Ryzhik [10], Eq. 4.337.2, Page 574 , and simplifying, we have

$$
E(X)=-e^{\lambda} E i(-\lambda), \lambda>0
$$

where $E i(z)$, known as the exponential-integral function, and is defined as follows:

$$
E i(z)=\gamma+\ln (-z)+\sum_{k=1}^{\infty} \frac{z^{k}}{k \cdot k !}, \quad z<0,
$$

and

$$
E i(z)=\gamma+\ln z+\sum_{k=1}^{\infty} \frac{z^{k}}{k \cdot k !}, \quad z>0,
$$

where $\gamma=-\psi(1) \approx 0.577216$ denotes the Euler's constant; (see, for example, Gradshteyn and Ryzhik [10], Eqs. 8.214.1 and 8.214.2, Page 927, Abramowitz and Stegun [1], Ch. 5, Page 228, and Oldham et al. [14], Ch. 37, Page 375, among others). In (2.7), taking $\lambda=1$, and noting that, since $-e E i(-1) \approx 0.59635$, known as the Gompertz constant, see, for example Finch [6] and Mezo [12], the 1 st moment for $k=1$ and $\lambda=1$ is given by

$$
E(X)=0.59635
$$

For a detailed treatment of properties of Chen's two-parameter exponential power life-testing distribution, we refer the interested readers to Chen [5].

\section{Characterization Results}

In this section, we provide our proposed characterizations of Chen's two-parameter exponential power life-testing distribution, with pdf (2.1) and cdf (2.2), by truncated moment. For this, we will need the following assumption and lemmas. 


\section{Assumption and Lemmas}

Assumptions 3.1. Suppose the random variable $X$ is absolutely continuous with the cumulative distribution function $F(x)$ and the probability density function $f(x)$. We assume that $\omega=\inf \{x \mid F(x)>0\}$, and $\delta=\sup \{x \mid F(x)<1\}$. We also assume that $f(x)$ is a differentiable for all $x$, and $E(X)$ exists.

Lemma 3.1. Under the Assumption 3.1, if $E(X \mid X \leq x)=g(x) \tau(x)$, where $\tau(x)=\frac{f(x)}{F(x)}$ and $g(x)$ is a continuous differentiable function of $x$ with the condition that $\int_{0}^{x} \frac{u-g^{\prime}(u)}{g(u)} d u$ is finite for $x>0$, then $f(x)=c e^{\int_{0}^{x} \frac{u-g^{\prime}(u)}{g(u)} d u}$, where $c$ is a constant determined by the condition $\int_{0}^{\infty} f(x) d x=1$.

Proof. Suppose that $E(X \mid X \leq x)=g(x) \tau(x)$. Then, since $E(X \mid X \leq x)=\frac{\int_{0}^{x} u f(u) d u}{F(x)}$ and $\tau(x)=\frac{f(x)}{F(x)}$, we have $g(x)=\frac{\int_{0}^{x} u f(u) d u}{f(x)}$, that is, $\int_{0}^{x} u f(u) d u=f(x) g(x)$.

Differentiating both sides of the above equation with respect to $x$, we obtain

$$
x f(x)=f^{\prime}(x) g(x)+f(x) g^{\prime}(x) .
$$

From the above equation, we obtain

$$
\frac{f^{\prime}(x)}{f(x)}=\frac{x-g^{\prime}(x)}{g(x)}
$$

On integrating the above equation with respect to $x$, we have

$$
f(x)=c e^{\int_{0}^{x} \frac{u-g^{\prime}(u)}{g(u)} d u},
$$

where $c$ is obtained by the condition $\int_{0}^{\infty} f(x) d x=1$. This completes the proof of Lemma 3.1. 
Lemma 3.2. Under the Assumption 3.1, if $E(X \mid X \geq x)=\tilde{g}(x) r(x)$, where $r(x)=\frac{f(x)}{1-F(x)}$ and $\tilde{g}(x)$ is a continuous differentiable function of $x$ with the condition that $\int_{x}^{\infty} \frac{u+[\tilde{g}(u)]^{\prime}}{\tilde{g}(u)} d u$ is finite for $x>0$, then $f(x)=c e^{-\int_{0}^{u+[\tilde{g}(u)]^{\prime}} \frac{\tilde{g}(u)}{g}}$, where $c$ is a constant determined by the condition $\int_{0}^{\infty} f(x) d x=1$.

Proof. Suppose that $E(X \mid X \geq x)=\tilde{g}(x) r(x)$. Then, since $E(X \mid X \geq x)=\frac{\int_{x}^{\infty} u f(u) d u}{1-F(x)}$ and $r(x)=\frac{f(x)}{1-F(x)}$, we have $\tilde{g}(x)=\frac{\int_{x}^{\infty} u f(u) d u}{f(x)}$, that is, $\int_{x}^{\infty} u f(u) d u=f(x) \tilde{g}(x)$

Differentiating the above equation with respect to respect to $x$, we obtain

$$
-x f(x)=f^{\prime}(x) \tilde{g}(x)+f(x)[\tilde{g}(x)]^{\prime}
$$

From the above equation, we obtain

$$
\frac{f^{\prime}(x)}{f(x)}=-\frac{x+[\tilde{g}(x)]^{\prime}}{\tilde{g}(x)}
$$

On integrating the above equation with respect to $x$, we have

$$
f(x)=c e^{-\int_{0}^{x} \frac{u+[\tilde{g}(u)]^{\prime}}{\tilde{g}(u)} d u},
$$

where $c$ is obtained by the condition $\int_{0}^{\infty} f(x) d x=1$. This completes the proof of Lemma 3.2. 
Theorem 3.1. If the random variable $X$ satisfies the Assumption 3.1 with $\omega=0$ and $\delta=\infty$, then $E(X \mid X \leq x)=g(x) \frac{f(x)}{F(x)}$, where

$$
g(x)=\frac{-x}{\lambda k x^{k-1} e^{x^{k}}}+\frac{e^{\lambda} \sum_{j=0}^{\infty} \frac{(-1)^{j}}{j !} \frac{1}{k} \frac{1}{j^{1 / k}} \Gamma_{j x^{k}}\left(\frac{1}{k}\right)}{\lambda k x^{k-1} e^{x^{k}} e^{\lambda\left(1-e^{x^{k}}\right)}}
$$

if and only if $X$ has the distribution with the pdf (2.1).

Proof. Suppose that $E(X \mid X \leq x)=g(x) \frac{f(x)}{F(x)}$. Then, since $E(X \mid X \leq x)=\frac{\int_{0}^{x} u f(u) d u}{F(x)}$, we have $g(x)=\frac{\int_{0}^{x} u f(u) d u}{f(x)}$. Now, if the random variable $X$ satisfies the Assumption 3.1 and has the distribution with the pdf (2.1), then we have

$$
\begin{aligned}
g(x)=\frac{\int_{0}^{x} u f(u) d u}{f(x)}=\frac{-\left.u(1-F(u))\right|_{0} ^{x}}{f(x)}+\frac{\int_{0}^{x}(1-F(u)) d u}{f(x)} \\
=\frac{-x}{\lambda k x^{k-1} e^{x^{k}}}+\frac{\int_{0}^{x} e^{\lambda\left(1-e^{u^{k}}\right)} d u}{\lambda k x^{k-1} e^{x^{k}} e^{\lambda\left(1-e^{x^{k}}\right)}} \\
=\frac{-x}{\lambda k x^{k-1} e^{x^{k}}}+\frac{e^{\lambda} \sum_{j=0}^{\infty} \int_{0}^{x} \frac{(-1)^{j} \lambda^{j} e^{j u^{k}}}{j !} d u}{\lambda k x^{k-1} e^{x^{k}} e^{\lambda\left(1-e^{x^{k}}\right)}} \\
=\frac{-x}{\lambda k x^{k-1} e^{x^{k}}}+\frac{e^{\lambda} \sum_{j=0}^{\infty} \frac{(-1)^{j}}{j !} \frac{1}{k} \frac{1}{j^{1 / k}} \Gamma_{j x^{k}}\left(\frac{1}{k}\right)}{\lambda k x^{k-1} e^{x^{k}} e^{\lambda\left(1-e^{x^{k}}\right)}}
\end{aligned}
$$

where $\Gamma_{x}(n)=\int_{0}^{x} u^{n-1} e^{u} d u$. 
Conversely, suppose that

$$
g(x)=\frac{-x}{\lambda k x^{k-1} e^{x^{k}}}+\frac{e^{\lambda} \sum_{j=0}^{\infty} \frac{(-1)^{j}}{j !} \frac{1}{k} \frac{1}{j^{1 / k}} \Gamma_{j x^{k}}\left(\frac{1}{k}\right)}{\lambda k x^{k-1} e^{x^{k}} e^{\lambda\left(1-e^{k^{k}}\right)}},
$$

where $\Gamma_{x}(n)=\int_{0}^{x} u^{n-1} e^{u} d u$.

Then, using Lemma 3.1, differentiating $g(x)$ with respect to $x$, and simplifying, we have

$$
\begin{gathered}
g^{\prime}(x) \\
=x-\left[\frac{-x}{\lambda k x^{k-1} e^{x^{k}}}+\frac{e^{\lambda} \sum_{j=0}^{\infty} \frac{(-1)^{j}}{j !} \frac{1}{k} \frac{1}{j^{1 / k}} \Gamma_{j x^{k}}\left(\frac{1}{k}\right)}{\lambda k x^{k-1} e^{x^{k}} e^{\lambda\left(1-e^{x^{k}}\right)}}\right]\left(\frac{k-1}{x}+k x^{k-1}-k x^{k-1} e^{x^{k}}\right) \\
=x-g(x)\left(\frac{k-1}{x}+k x^{k-1}-k x^{k-1} e^{x^{k}}\right),
\end{gathered}
$$

from which we obtain

$$
\frac{x-g^{\prime}(x)}{g(x)}=\frac{k-1}{x}+k x^{k-1}-k x^{k-1} e^{x^{k}} .
$$

Since, by Lemma 3.1, we have

$$
\frac{x-g^{\prime}(x)}{g(x)}=\frac{f^{\prime}(x)}{f(x)},
$$

it follows that

$$
\frac{f^{\prime}(x)}{f(x)}=\frac{k-1}{x}+k x^{k-1}-\lambda k x^{k-1} e^{x^{k}}
$$

On integrating the above expression with respect to $x$ and simplifying, we obtain

$$
\ln f(x)=\ln \left(c x^{k-1} e^{x^{k}} e^{-\lambda e^{x^{k}}}\right)
$$

or,

$$
f(x)=c x^{k-1} e^{x^{k}} e^{-\lambda e^{x^{k}}}
$$


where $c$ is the normalizing constant to be determined. Thus, on integrating the above equation with respect to $x$ from $x=0$ to $x=\infty$, and using the condition $\int_{0}^{\infty} f(x) d x=1$, we easily obtain

$$
c=\lambda k e^{\lambda},
$$

and, hence, we have

$$
f(x)=\lambda k x^{k-1} e^{x^{k}} e^{\lambda\left(1-e^{x^{k}}\right)}, k>0, \lambda>0
$$

which is the required pdf (2.1). This completes the proof of Theorem 3.1.

Theorem 3.2. If the random variable $X$ satisfies the Assumption 3.1 with $\omega=0$ and $\delta=\infty$, then $E(X \mid X \geq x)=\tilde{g}(x) \frac{f(x)}{1-F(x)}$, where

$$
\tilde{g}(x)=\frac{x}{\lambda k x^{k-1} e^{x^{k}}}+\frac{e^{\lambda} \sum_{j=0}^{\infty} \frac{(-1)^{j}}{j !} \frac{\lambda^{j}}{k} \frac{1}{j^{1 / k}} \Gamma_{j x^{k}}^{*}\left(\frac{1}{k}\right)}{\lambda k x^{k-1} e^{x^{k}} e^{\lambda\left(1-e^{k^{k}}\right)}},
$$

where $\Gamma_{x}^{*}(n)=\int_{x}^{\infty} u^{n-1} e^{u} d u$, if and only if $X$ has the distribution with the pdf (2.1).

Proof. Suppose that $E(X \mid X \geq x)=\tilde{g}(x) \frac{f(x)}{1-F(x)}$. Then, since $E(X \mid X \geq x)=\frac{\int_{x}^{\infty} u f(u) d u}{1-F(x)}$, we have $\tilde{g}(x)=\frac{\int_{x}^{\infty} u f(u) d u}{f(x)}$. Now, if the random variable $X$ satisfies the Assumptions 3.1 and has the distribution with the pdf as given in (2.1), then we have

$$
\begin{gathered}
\tilde{g}(x)=\frac{\int_{x}^{\infty} u f(u) d u}{f(x)} \\
=\frac{-\left.u(1-F(u))\right|_{x} ^{\infty}}{f(x)}+\frac{\int_{x}^{\infty}(1-F(u)) d u}{f(x)} \\
=\frac{x}{\lambda k x^{k-1} e^{x^{k}}}+\frac{\int_{x}^{\infty} e^{\lambda\left(1-e^{u^{k}}\right)} d u}{\lambda k x^{k-1} e^{x^{k}} e^{\lambda\left(1-e^{x^{k}}\right)}}
\end{gathered}
$$




$$
\begin{gathered}
=\frac{x}{\lambda k x^{k-1} e^{x^{k}}}+\frac{e^{\lambda} \sum_{j=0}^{\infty} \int_{x}^{\infty} \frac{(-1)^{j} \lambda^{j} e^{j u^{k}}}{j !} d u}{\lambda k x^{k-1} e^{x^{k}} e^{\lambda\left(1-e^{x^{k}}\right)}} \\
=\frac{x}{\lambda k x^{k-1} e^{x^{k}}}+\frac{e^{\lambda} \sum_{j=0}^{\infty} \frac{(-1)^{j}}{j !} \frac{\lambda^{j}}{k} \frac{1}{j^{1 / k}} \Gamma_{j x^{k}}^{*}\left(\frac{1}{k}\right)}{\lambda k x^{k-1} e^{x^{k}} e^{\lambda\left(1-e^{x^{k}}\right)}}
\end{gathered}
$$

where $\Gamma_{x}^{*}(n)=\int_{x}^{\infty} u^{n-1} e^{u} d u$. Note that $\sum_{j=0}^{\infty} \frac{(-1)^{j}}{j !} \frac{\lambda^{j}}{k} \frac{1}{j^{1 / k}} \Gamma_{j x^{k}}^{*}\left(\frac{1}{k}\right)$ is convergent for all $x$.

Conversely, suppose that $\tilde{g}(x)=\frac{x}{\lambda k x^{k-1} e^{x^{k}}}+\frac{e^{\lambda} \sum_{j=0}^{\infty} \frac{(-1)^{j}}{j !} \frac{\lambda^{j}}{k} \frac{1}{j^{1 / k}} \Gamma_{j x^{k}}^{*}\left(\frac{1}{k}\right)}{\lambda k x^{k-1} e^{x^{k}} e^{\lambda\left(1-e^{x^{k}}\right)}}$, where $\Gamma_{x}^{*}(n)=\int_{x}^{\infty} u^{n-1} e^{u} d u$

Then, using Lemma 3.2, differentiating $\tilde{g}(x)$ with respect to $x$, and simplifying, we have

$$
\begin{gathered}
(\tilde{g}(x))^{\prime} \\
\quad=-x-\left[\frac{x}{\lambda k x^{k-1} e^{x^{k}}}+\frac{e^{\lambda} \sum_{j=0}^{\infty} \frac{(-1)^{j}}{j !} \frac{\lambda^{j}}{k} \frac{1}{j^{1 / k}} \Gamma_{j x^{k}}^{*}\left(\frac{1}{k}\right)}{\left.\lambda k x^{k-1} e^{x^{k}} e^{\lambda\left(1-e^{x^{k}}\right)}\right]\left(\frac{k-1}{x}+k x^{k-1}-k x^{k-1} e^{x^{k}}\right)}\right. \\
=-x-\tilde{g}(x)\left(\frac{k-1}{x}+k x^{k-1}-k x^{k-1} e^{x^{k}}\right),
\end{gathered}
$$

from which we obtain

$$
\frac{x+(\tilde{g}(x))^{\prime}}{\tilde{g}(x)}=-\left(\frac{k-1}{x}+k x^{k-1}-k x^{k-1} e^{x^{k}}\right) .
$$

Since, by Lemma 3.2, we have 


$$
\frac{f^{\prime}(x)}{f(x)}=-\frac{x+[\tilde{g}(x)]^{\prime}}{\tilde{g}(x)},
$$

it follows that

$$
\frac{f^{\prime}(x)}{f(x)}=\frac{k-1}{x}+k x^{k-1}-k x^{k-1} e^{x^{k}}
$$

On integrating the above expression with respect to $x$ and simplifying, we obtain

$$
\ln f(x)=\ln \left(c x^{k-1} e^{x^{k}} e^{-\lambda e^{x^{k}}}\right)
$$

or,

$$
f(x)=c x^{k-1} e^{x^{k}} e^{-\lambda e^{k^{k}}}
$$

where $c$ is the normalizing constant to be determined. Thus, on integrating the above equation with respect to $x$ from $x=0$ to $x=\infty$, and using the condition $\int_{0}^{\infty} f(x) d x=1$, we easily obtain

$$
c=\lambda k e^{\lambda} \text {, }
$$

and, hence, we have

$$
f(x)=\lambda k x^{k-1} e^{x^{k}} e^{\lambda\left(1-e^{x^{k}}\right)}, k>0, \lambda>0
$$

which is the required pdf (2.1). This completes the proof of Theorem 3.2.

\section{Conclusion}

In this paper, we have considered the two-parameter exponential power life-testing distribution introduced by Chen [5], and provided its characterizations by truncated moment method. We hope the findings of the paper will be quite useful for the practitioners in various fields of sciences. 


\section{ACKNOWLEDGEMENT}

Authors are thankful to the referees and editor-in-chief of the journal for their valuable comments and suggestions, which improved the presentation of this paper greatly. The first author, M. Shakil, is grateful to Miami Dade College for giving him the opportunity to be of service to this institution, without which it would have been impossible to conduct his research. Also, this article was partially completed while the third author, B. M. Golam Kibria, was on sabbatical leave (Fall 2017). He is grateful to Florida International University for awarding him the sabbatical leave which gave him excellent research facilities.

Declarations: We confirm that none of the authors have any competing interests in the manuscript.

\section{References}

[1] Abramowitz, M., and Stegun, I. A. (1970). Handbook of Mathematical Functions with Formulas, Graphs, and Mathematical Tables. National Bureau of Standards, Washington, D. C.

[2] Ahsanullah, M. (2017), Characterizations of Univariate Continuous Distributions. Atlantis Press, Paris, France.

[3] Ahsanullah, M., Kibria, B. M. G., and Shakil, M. (2014). Normal and Student's $t$ Distributions and Their Applications. Atlantis Press, Paris, France.

[4] Chen, Z. (1999). Statistical inference about the shape parameter of the exponential power distribution. Statistical Papers, Vol. 40(4), 459 - 468.

[5] Chen, Z. (2000). A new two-parameter lifetime distribution with bathtub shape or increasing failure rate function. Statistics \& Probability Letters, 49(2), $155-161$.

[6] Finch, S. R. (2003). Mathematical Constants. Cambridge University Press, Cambridge, UK.

[7] Galambos, J., and Kotz, S. (1978). Characterizations of probability distributions. A unified approach with an emphasis on exponential and related models, Lecture Notes in Mathematics, 675, Springer, Berlin.

[8] Glänzel, W. (1987). A characterization theorem based on truncated moments and its application to some distribution families, Mathematical Statistics and Probability Theory (Bad Tatzmannsdorf, 1986), Vol. B, Reidel, Dordrecht, 75 - 84.

[9] Glänzel, W., Telcs, A., and Schubert, A. (1984). Characterization by truncated moments and its application to Pearson-type distributions, Z. Wahrsch. Verw. Gebiete, 66, $173-183$.

[10] Gradshteyn, I. S., and Ryzhik, I. M. (1980). Table of integrals, series, and products, Academic Press, Inc., San Diego, California, USA.

[11] Kotz, S., and Shanbhag, D. N. (1980). Some new approaches to probability distributions. Advances in Applied Probability, 12, 903 - 921.

[12] Mezo, I. (2014). "Gompertz constant, Gregory coefficients and a series of the logarithm function". Journal of Analysis and Number Theory, 2 (2), 33 - 36. 
[13] Leemis, L. M. (1986). Lifetime distribution identities. Reliability, IEEE Transactions on, 35(2), 170 - 174.

[14] Oldham, K. B., Myland, J., and Spanier, J. (2009). An Atlas of Functions with Equator, the Atlas Function Calculator. Springer, New York, USA.

[15] Rajarshi, S., and Rajarshi, M. B. (1988). Bathtub distributions: A review. Communications in Statistics-Theory and Methods, 17(8), 2597 - 2621.

[16] Smith, R. M., and Bain, L. J. (1975). An exponential power life-testing distribution. Communications in Statistics-Theory and Methods, 4(5), 469 481. 